\title{
Subdivided Claws and the Clique-Stable Set Separation Property
}

\author{
Maria Chudnovsky*and Paul Seymour ${ }^{\dagger}$ \\ Princeton University, Princeton, NJ 08544
}

December 6, 2019; revised December 19, 2019

\begin{abstract}
Let $\mathcal{C}$ be a class of graphs closed under taking induced subgraphs. We say that $\mathcal{C}$ has the clique-stable set separation property if there exists $c \in \mathbb{N}$ such that for every graph $G \in \mathcal{C}$ there is a collection $\mathcal{P}$ of partitions $(X, Y)$ of the vertex set of $G$ with $|\mathcal{P}| \leq|V(G)|^{c}$ and with the following property: if $K$ is a clique of $G$, and $S$ is a stable set of $G$, and $K \cap S=\emptyset$, then there is $(X, Y) \in \mathcal{P}$ with $K \subseteq X$ and $S \subseteq Y$. In $1991 \mathrm{M}$. Yannakakis conjectured that the class of all graphs has the clique-stable set separation property, but this conjecture was disproved by M. Göös in 2014. Therefore it is now of interest to understand for which classes of graphs such a constant $c$ exists. In this paper we define two infinite families $\mathcal{S}, \mathcal{K}$ of graphs and show that for every $S \in \mathcal{S}$ and $K \in \mathcal{K}$, the class of graphs with no induced subgraph isomorphic to $S$ or $K$ has the clique-stable set separation property.
\end{abstract}

\section{Introduction}

All graphs in this paper are finite and simple. Let $G$ be a graph. A clique in $G$ is a set of pairwise adjacent vertices, and a stable set is a set of pairwise non-adjacent vertices. Let $\mathcal{C}$ be a class of graphs closed under taking induced subgraphs. We say that $\mathcal{C}$ has the clique-stable set separation property if there exists $c \in \mathbb{N}$ such that for every graph $G \in \mathcal{C}$ there is a collection $\mathcal{P}$ of partitions $(X, Y)$ of the vertex set of $G$ with $|\mathcal{P}| \leq|V(G)|^{c}$ and with the following property: if $K$ is a clique of $G$, and $S$ is a stable set of $G$, and $K \cap S=\emptyset$, then there is $(X, Y) \in \mathcal{P}$ with $K \subseteq X$ and $S \subseteq Y$. This property plays an important role in a large variety of fields: communication complexity, combinatorial optimization, constraint satisfaction and others (for a comprehensive survey of these connections see [3]).

In 1991 Mihalis Yannakakis conjectured that the class of all graphs has the clique-stable set separation property [5], but this conjecture was disproved by Mika Göös in 2014 [2]. Therefore it is now of interest to understand for which classes of graphs such a constant $c$ exists; our main result falls into that category.

Let $G$ be a graph and let $X, Y$ be disjoint subsets of $V(G)$. We denote by $G[X]$ the subgraph of $G$ induced by $X$, by $N(X)$ the set of all vertices of $V(G) \backslash X$ with a neighbor in $X$, and by $N[X]$ the set $N(X) \cup X$. We say that $X$ is complete to $Y$ if every vertex of $X$ is adjacent to every vertex of $Y$, and that $X$ is anticomplete to $Y$ if every vertex of $X$ is non-adjacent to every vertex of $Y$. We say

\footnotetext{
${ }^{*}$ This material is based upon work supported in part by the U. S. Army Research Office under grant number W911NF-16-1-0404, and by NSF grant DMS-1763817.

${ }^{\dagger}$ Partially supported by NSF grant DMS-1800053 and AFOSR grant A9550-19-1-0187.
} 
that $X$ and $Y$ are matched if every vertex of $X$ has exactly one neighbor in $Y$, and every vertex of $Y$ has exactly one neighbor in $X$ (and therefore $|X|=|Y|$ ). For a graph $H$, we say that $G$ is $H$-free if no induced subgraph of $G$ is isomorphic to $H$.

Next we define two types of graphs. Let $p, q \in \mathbb{N}$. We define the graph $F_{S}^{p, q}$ as follows:

- $V\left(F_{S}^{p, q}\right)=K \cup S_{1} \cup S_{2} \cup S_{3}$ where $K$ is a clique, $S_{1}, S_{2}, S_{3}$ are stable sets, and the sets $K, S_{1}, S_{2}, S_{3}$ are pairwise disjoint;

- $|K|=\left|S_{1}\right|=p$, and $K$ and $S_{1}$ are matched;

- $\left|S_{2}\right|=\left|S_{3}\right|=q$, and $S_{2}$ and $S_{3}$ are matched;

- $K$ is complete to $S_{2}$;

- there are no other edges in $F_{S}^{p, q}$.

The graph $F_{K}^{p, q}$ is obtained from $F_{S}^{p, q}$ by making all pairs of vertices of $S_{3}$ adjacent.
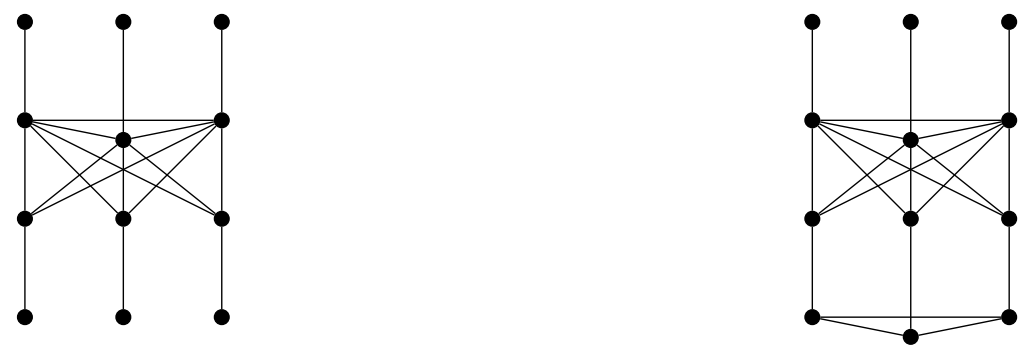

Figure 1: The graphs $F_{S}^{3,3}$ and $F_{K}^{3,3}$

Let $\mathcal{F}^{p, q}$ be the class of all graphs that are both $F_{S}^{p, q}$-free and $F_{K}^{p, q}$-free. We can now state our main result:

\subsection{For all $p, q>0$ the class $\mathcal{F}^{p, q}$ has the clique-stable set separation property.}

Since the clique-stable set separation property is preserved under taking complements, we immediately deduce:

1.2 For all $p, q>0$ the class of graphs whose complements are in $\mathcal{F}^{p, q}$ has the clique-stable set separation property.

\section{The Proof}

In this section we prove 1.1. The idea of the proof comes from [1. Let $G \in \mathcal{F}^{p, q}$. Define $\mathcal{P}_{1}$ to be the set of all partitions $(N[X], V(G) \backslash N[X])$ and $(N(X), V(G) \backslash N(X))$ where $X$ is a subset of $V(G)$ with $|X|<p$. Clearly $\left|\mathcal{P}_{1}\right| \leq 2|V(G)|^{p}$.

Write $R=R(q, q)$ to mean the smallest positive integer $R$ such that every 2-coloring of the edges of the complete graph on $R$ vertices contains a monochromatic complete graph on $q$ vertices. Ramsey's Theorem [4] implies: 
$2.1 R(q, q) \leq 2^{2 q}$.

For $a, b \in \mathbb{N}$ let the graph $F_{a, b}$ be defined as follows:

- $V\left(F_{a, b}\right)=K_{1} \cup S_{1} \cup S_{2} \cup W$ where $K_{1}$ is a clique, $S_{1}, S_{2}$ are stable sets, and the sets $K_{1}, S_{1}, S_{2}, W$ are pairwise disjoint;

- $\left|K_{1}\right|=\left|S_{1}\right|=a$, and $K_{1}$ and $S_{1}$ are matched;

- $\left|S_{2}\right|=|W|=b$, and $S_{2}$ and $W$ are matched;

- $K_{1}$ is complete to $S_{2}$;

- there is no restriction on the adjacency of pairs of vertices of $W$;

- there are no other edges in $F_{a, b}$.

From the definition of $R$ we immediately deduce:

$2.2 G$ is $F_{p, R}$-free.

For every triple $X=\left(K_{1}, S_{1}, S_{2}\right)$ of pairwise disjoint non-emtpy subsets of $V(G)$ such that $\left|K_{1}\right|=$ $\left|S_{1}\right|=p$ and $\left|S_{2}\right|<R$ we define the partition $P_{X}$ of $V(G)$ as follows. Let $Z$ be the set of all vertices of $G$ that are anticomplete to $K_{1} \cup S_{1}$. Let $A_{X}$ be the set of all vertices $v$ of $G$ such that

- either $v \in K_{1}$, or $v$ is complete to $K_{1}$, and

- either $v$ has a neighbor in $S_{1}$, or $v$ has a neighbor in $Z \backslash N\left(S_{2}\right)$.

Note that $A_{X}$ is disjoint from $S_{1} \cup Z$. Define $P_{X}=\left(A_{X}, V(G) \backslash A_{X}\right)$, and let $\mathcal{P}_{2}$ be the set of all such partitions $P_{X}$. Since $\left|K_{1} \cup S_{1} \cup S_{2}\right| \leq 2 p+R-1$, and since by $2.1 R \leq 2^{2 q}$, we deduce that $\left|\mathcal{P}_{2}\right|<|V(G)|^{2 p+2^{2 q}}$.

In order to complete the proof of 1.1 we will prove the following:

2.3 For every clique $K$ and stable set $S$ of $G$ such that $K \cap S=\emptyset$, there exists $(X, Y) \in \mathcal{P}_{1} \cup \mathcal{P}_{2}$ with $K \subseteq X$ and $S \subseteq Y$.

Proof: Let $K$ and $S$ be as in the statement of 2.3 .

(1) We may assume that $K$ is a maximal clique of $G$, and $S$ is a maximal stable set of $G$.

Let $K^{\prime}$ be a maximal clique of $G$ with $K \subseteq K^{\prime}$, and let $S^{\prime}$ be a maximal stable set of $G$ with $S \subseteq S^{\prime}$. If $K^{\prime} \cap S^{\prime}=\emptyset$, then the existence of the desired partition for $K, S$ follows from the existence of such a partition for $K^{\prime}, S^{\prime}$; thus we may assume that $K^{\prime} \cap S^{\prime} \neq \emptyset$. Since $K^{\prime}$ is a clique and $S^{\prime}$ is a stable set, it follows that $\left|K^{\prime} \cap S^{\prime}\right|=1$, say $K^{\prime} \cap S^{\prime}=\{v\}$. But now the partitions $(N[\{v\}], V(G) \backslash N[\{v\}])$ and $(N(\{v\}), V(G) \backslash N(\{v\}])$ are both in $\mathcal{P}_{1}$, and at least one of them has the desired property. This proves (1). 
In view of (11) from now on we assume that $K$ is a maximal clique of $G$, and $S$ is a maximal stable set of $G$. Consequently every vertex of $K$ has a neighbor in $S$. Let $S_{1}^{\prime} \subseteq S$ be a minimal subset of $S$ such that every vertex of $K$ has a neighbor in $S_{1}^{\prime}$. It follows from the minimality of $S_{1}^{\prime}$ that there is a subset $K_{1}^{\prime}$ of $K$ such that $S_{1}^{\prime}$ and $K_{1}^{\prime}$ are matched. If $\left|S_{1}^{\prime}\right|<p$, then the partition $\left(N\left(S_{1}^{\prime}\right), V(G) \backslash N\left(S_{1}^{\prime}\right)\right) \in \mathcal{P}_{1}$ has the desired property, so we may assume that $\left|S_{1}^{\prime}\right| \geq p$.

Let $S_{1}$ be a subset of $S_{1}^{\prime}$ with $\left|S_{1}\right|=p$, and let $K_{1}=N\left(S_{1}\right) \cap K_{1}^{\prime}$. Then $S_{1}$ and $K_{1}$ are matched, and so $\left|K_{1}\right|=p$. Let $Z$ be the set of vertices of $G$ that are anticomplete to $S_{1} \cup K_{1}$. Then $S_{1}^{\prime} \backslash S_{1} \subseteq Z \cap S$, and in particular every vertex of $K$ has a neighbor either in $S_{1}$ or in $Z \cap S$. Let $S^{\prime}$ be the subset of vertices of $S \backslash S_{1}$ that are complete to $K_{1}$. Note that $S^{\prime} \cap Z=\emptyset$. Let $S_{2}$ be a minimal subset of $S^{\prime}$ such that $N\left(S_{2}\right) \cap Z=N\left(S^{\prime}\right) \cap Z$. It follows from the minimality of $S_{2}$ that there is a subset $W \subseteq Z \cap N\left(S^{\prime}\right)$ such that $W$ and $S_{2}$ are matched. Observe that $G\left[K_{1} \cup S_{1} \cup S_{2} \cup W\right]$ is isomorphic to $F_{p,\left|S_{2}\right|}$ (with $K_{1}, S_{1}, S_{2}, W$ as in the definition of $F_{a, b}$ ). It follows from 2.2 that $\left|S_{2}\right|<R$.

Let $X=\left(K_{1}, S_{1}, S_{2}\right)$. We claim that the partition $P_{X} \in \mathcal{P}_{2}$ has the desired property for the pair $K, S$. Recall that $P_{X}=\left(A_{X}, V(G) \backslash A_{X}\right)$, where $A_{X}$ is the set of all vertices $v$ of $G$ such that

- either $v \in K_{1}$, or $v$ is complete to $K_{1}$, and

- either $v$ has a neighbor in $S_{1}$, or $v$ has a neighbor in $Z \backslash N\left(S_{2}\right)$.

We need to show that $K \subseteq A_{X}$, and $S \cap A_{X}=\emptyset$.

(2) $K \subseteq A_{X}$.

Let $k \in K$. Clearly either $k \in K_{1}$ or $k$ is complete to $K_{1}$. Moreover, $k$ has a neighbor in $S_{1}^{\prime}$, and $S_{1}^{\prime} \subseteq S_{1} \cup(Z \cap S)$. Since $S$ is a stable set, it follows that $Z \cap S \subseteq Z \backslash N\left(S_{2}\right)$, and thus $k$ has a neighbor either in $S_{1}$, or in $Z \backslash N\left(S_{2}\right)$. This proves (2).

$$
S \cap A_{X}=\emptyset .
$$

Suppose that $s \in S \cap A_{X}$. Then $s \notin K_{1}$; therefore $s$ is complete to $K_{1}$, and so $s \in S^{\prime}$. Since $S$ is a stable set, it follows that $s$ is anticomplete to $S_{1}$, and therefore $s$ has a neighbor in $Z \backslash N\left(S_{2}\right)$. But $N\left(S^{\prime}\right) \cap Z=N\left(S_{2}\right) \cap Z$, a contradiction. This proves (3).

Now 2.3 follows from (2) and (3).

This completes the proof of 1.1 .

\section{Acknowledgment}

This work was done during the Structural Graph Theory Downunder Matrix Program, that was held in 2019 at the Creswick Campus of the University of Melbourne. The authors express their gratitude to the Matrix Center for the funding it provided and the use of its facilities, and to the organizers of the program for the invitation. 


\section{References}

[1] T. Abrishami, M. Chudnovsky and M. Pilipczuk, "Containers for the maximum weight stable set problem", in preparation.

[2] M. Göös, "Lower bounds for clique vs. independent Set", Proc. 56th Foundations of Computer Science (FOCS), 2015: 1066-1077.

[3] A. Lagoutte, "Interactions entre les cliques et les stables dans un graphe", PhD thesis, ENS de Lyon, 2015.

[4] F.P. Ramsey, "On a problem of formal logic", Proc. London Math. Soc., 30, 1930: 264-286.

[5] M. Yannakakis, "Expressing combinatorial optimization problems by linear programs", J. Comput. Syst.. Sci, 43 (3), 1991: 441-466. 